\title{
Análisis citotaxonómico del complejo Alstroemeria diluta E. Bayer (Alstroemeriaceae) de Chile
}

\section{Cytotaxonomic analysis in the Alstroemeria diluta E. Bayer (Alstroemeriaceae) complex from Chile}

\author{
Carlos M. Baeza ${ }^{1 *}$, Víctor L. Finot ${ }^{2}$, Eduardo Ruiz ${ }^{1}$, Pedro Carrasco ${ }^{1}$, Patricio Novoa $^{3}$ \& Marcelo \\ ROSAS $^{4}$
}

${ }^{1}$ Facultad de Ciencias Naturales y Oceanográficas, Departamento de Botánica, Casilla 160-C, Universidad de Concepción, Concepción, Chile.

2Departamento de Producción Animal, Facultad de Agronomía, Universidad de Concepción, Chillán. ${ }^{3} J a r d i ́ n$ Botánico de Viña del Mar, Corporación Nacional Forestal, Camino El Olivar 305, Viña del Mar.

${ }^{4}$ Programa de Doctorado en Sistemática y Biodiversidad, Departamento de Botánica, Casilla 160-C, Universidad de Concepción, Concepción, Chile.

*cbaeza@udec.cl

\begin{abstract}
RESUMEN
Alstroemeria L. es uno de los géneros de plantas chilenas más diversos y con un enorme potencial ornamental. Está representado por 35 especies, la mayoría distribuida entre los $28-38^{\circ} \mathrm{S}$ en la zona mediterránea de Chile central. Se reconocen 24 complejos, de los cuales 18 presentan problemas de conservación. Uno de estos complejos es Alstroemeria diluta E. Bayer. con dos subespecies: subsp. diluta y subsp. chrysantha Bayer. Alstroemeria diluta crece sólo en Chile; la subsp. diluta se distribuye desde los $34^{\circ} 15^{\prime}$ 'S, en la Región de O'Higgins, hasta Cauquenes (36 $06^{\circ}$ 'S), en la Región

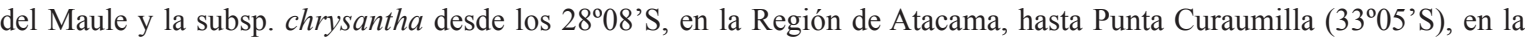
Región de Valparaíso. Se realizó un estudio comparativo del cariotipo de cuatro poblaciones de A. diluta subsp. diluta y tres poblaciones de $A$. diluta subsp. chrysantha. Las siete poblaciones analizadas presentaron un cariotipo asimétrico, con un $2 \mathrm{n}=2 \mathrm{x}=16$ cromosomas, y con la misma fórmula cariotípica: $3 \mathrm{~m}+1 \mathrm{sm}+1 \mathrm{st}+3 \mathrm{t}$. La arquitectura del cariotipo entre las subespecies es la misma. El diagrama de dispersión MCA vs CVCL mostró diferentes grupos entre las poblaciones de las dos subespecies y la longitud total diploide de los cromosomas (LTC) fue mayor en las poblaciones de la subsp. chrysantha. Los resultados obtenidos sugieren que las poblaciones distribuidas en la V Región de Valparaíso pertenecen a la subsp. diluta.
\end{abstract}

Palabras clave: Alstroemeria, asimetría, complejo de especies.

\begin{abstract}
Alstroemeria L., one of the most diverse genera of the Chilean flora and of high floricultural value, is represented by 35 species, most of them distributed between $28-38^{\circ} \mathrm{S}$ in the Mediterranean zone of Central Chile. There are 24 complexforming taxa, of which 18 have conservation problems. One of these complexes is Alstroemeria diluta E. Bayer. with two subspecies: subsp. diluta and subsp. chrysantha Bayer. Alstroemeria diluta grows only in Chile; subsp. diluta is distributed from $34^{\circ} 15^{\prime} \mathrm{S}$, Region of O'Higgins, to Cauquenes (36 $\left.06^{\circ} \mathrm{S}\right)$, Region of Maule, and subsp. chrysantha from $28^{\circ} 08^{\prime} \mathrm{S}$, Region of Atacama, to Punta Curaumilla ( $33^{\circ} 05^{\prime}$ S), Region of Valparaíso. A comparative karyotype study was carried out among four populations of $A$. diluta subsp. diluta and three populations of A. diluta subsp. chrysantha. The seven populations presented an asymmetric karyotype, with $2 \mathrm{n}=2 \mathrm{x}=16$ chromosomes, and with same karyotype formulae: $3 \mathrm{~m}$ $+1 \mathrm{sm}+1 \mathrm{st}+3 \mathrm{t}$. The architecture of the karyotype between the subspecies is the same. The scatter plot among MCA vs. CVCL shows different groupings between populations of the two subspecies, and the total chromosomes length (LTC) is highest in the populations of subsp. chrysantha. According to the results obtained, the populations growing in Valparaíso Region should be considered belong to subsp. diluta.
\end{abstract}

KEYwords: Alstroemeria, asymmetry, species complex. 


\section{INTRODUCCIÓN}

Alstroemeria es uno de los géneros más diversos de monocotiledóneas de la flora chilena, representado por 35 especies, la mayoría distribuidas entre los $28^{\circ} \mathrm{S}$ y $39^{\circ}$ S, en la zona central de Chile (Amigo \& Ramírez 1998, Muñoz \& Moreira 2003). Once especies de este género están constituidas por complejos de dos a cuatro unidades taxonómicas infraespecíficas (subespecies y variedades). La mayoría de estos complejos son endémicos, crecen en la zona mediterránea de Chile y presentan una distribución muy restringida (Bayer 1987, Muñoz \& Moreira 2003). Esta zona concentra el más alto porcentaje de la población humana del país, acompañado de una intensa intervención antrópica debido a la agricultura y actividades forestales como también por los asentamientos humanos, lo que constituye un riesgo para la conservación (Vergara et al. 2006).

Alstroemeria diluta E. Bayer es una especie que crece entre los $28^{\circ} \mathrm{S}$ y los $36^{\circ} \mathrm{S}$ (Muñoz \& Moreira 2003) y se reconocen en este complejo dos subespecies: subsp. diluta y subsp. chrysantha E. Bayer. Bayer (1987) caracteriza Alstroemeria diluta como una especie con las hojas de los tallos estériles extremadamente delgadas y por la presencia de anteras de color oliva. Reconoce dos subespecies que distingue por la coloración de los tépalos y por tener distribuciones muy diferentes. Con respecto al color de la flor Bayer (1987) caracteriza a la subsp. chrysantha por tener flores cuyo color principal es amarillo-dorado o rosa amarillento (Fig. 1 B) mientras indica que la subsp. diluta posee flores con color principal blanco a rosa-pálido sucio (Fig. 1 A). Con respecto a la distribución, la subsp. diluta se distribuye entre los $34^{\circ} 15^{\prime} \mathrm{S}$, en la Región de O’Higgins hasta Cauquenes (36 $06^{\circ}$ 'S), en la Región del Maule; mientras la subsp. chrysantha lo hace desde los $28^{\circ} 08^{\prime} \mathrm{S}$, en la Región de Atacama hasta Punta Curaumilla $\left(33^{\circ} 05^{\prime}\right.$ S), en la Región de Valparaíso (Muñoz \& Moreira 2003).

Baeza et al. (2010b) describen el cariotipo de una población de Alstroemeria diluta de la $\mathrm{V}$ Región de Valparaíso, indicando que presenta un cariotipo muy asimétrico.

Diversos problemas taxonómicos han sido esclarecidos gracias al estudio de los cromosomas en el género Alstroemeria, por ejemplo el estudio del cariotipo permitió reconocer una nueva subespecie, elevar a rango específico una subespecie, y describir una nueva especie para la ciencia en el complejo A. hookeri Loddiges (Cajas et al. 2009, Baeza et al. 2010 a, Baeza \& Ruiz 2011, Negritto et al. 2015). Una situación similar ocurrió en el complejo A. presliana Herbert, donde luego de realizar un estudio comparativo del cariotipo en 11 poblaciones se sugiere elevar a nivel específico $A$. presliana subsp. australis
Bayer, endémica de la cordillera de Nahuelbuta (Baeza et al. 2015a). Estudios citológicos han reforzado las diferencias entre las subespecies del complejo Alstroemeria ligtu L., y también han permitido sugerir reconocer una nueva subespecie dentro del complejo (Baeza et al. 2015b).

El objetivo de esta investigación fue, por lo tanto, caracterizar citotaxonómicamente las dos subespecies de Alstroemeria diluta a lo largo de su rango de distribución y entregar antecedentes que permitan clarificar el estatus taxonómico de las poblaciones de ambas subespecies.

\section{MATERIALES Y MÉTODO}

MATERIAL VEGETAL

Se recolectaron siete poblaciones de Alstroemeria diluta en todo su rango de distribución. Los ejemplares fueron depositados en el herbario de la Universidad de Concepción (CONC) y corresponden a los siguientes.

\section{A. diluta subsp. diluta}

Región de Valparaíso, Prov. Valparaíso. Viña del Mar. Jardín Botánico Nacional. Lote C. Lado sur quebrada Horquilla. 3202'30"S-71'29'43"O, 117 m, 2-XI-2013, Baeza 4390.

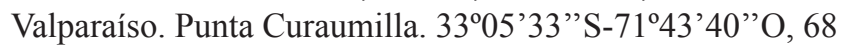
m, 2-XI-2013, Baeza 4391. Región del Maule. Prov. Talca. Río Claro. Km 229 Ruta Sur. 35¹5'30'S-71²8'54”'O, 181 m, 3-XI-2013, Baeza 4392. Río Claro, $300 \mathrm{~m}$ al norte del puente Río Claro, desde el sur, km 216, 190 m, 35²10'53'S71²3'19'”, 9-XI-2013, Baeza 4393.

\section{A. diluta subsp. chrysantha}

Región de Coquimbo, Prov. Elqui. Guanaqueros, al lado del camino. 30¹1'52"S-71'25'21"O, 33 m, 31-X-.2013, Baeza 4376. Coquimbo. Entre Las Tacas y Guanaqueros, km 440. 3008'13'S-71²2'04'”, 40 m, 7-X-2014, Baeza 4412. Prov. Limarí. Ovalle. Parque Nacional Fray Jorge, entrada antigua, 30³8'16"S-71'36 29"'O, 322 m, 8-X-2014, Baeza 4415.

La Figura 2 muestra la distribución de las poblaciones recolectadas y analizadas citológicamente.

\section{MÉTODO}

Raíces (1-2 cm de longitud) obtenidas a partir de material cultivado en invernadero fueron cortadas y pretratadas con una solución de 8-hidroxiquinolina $(2 \mathrm{mM})$ durante $24 \mathrm{~h}$ a $4{ }^{\circ} \mathrm{C}$. Posteriormente, se fijaron en (una mezcla fresca de) etanol/ácido acético (3:1) por $24 \mathrm{~h}$. Se realizaron aplastados de puntas de raíces efectuando previamente una hidrólisis ácida con $\mathrm{HCl} 0,5 \mathrm{~N}$ durante $17 \mathrm{~min}$ a $42^{\circ} \mathrm{C}$, para luego lavar el material y teñir la punta de raíz con orceína al $1 \%$. Los 
cromosomas fueron fotografiados en un microscopio Zeiss Axioskop con cámara de video incorporada. Las placas metafásicas fueron medidas con la ayuda del programa computacional "MicroMeasure 3.3" (Reeves 2001). A partir de 10 placas metafásicas por población analizada (5 individuos como mínimo), se procedió a construir el idiograma de cada subespecie en estudio. Para cada población analizada se calcularon los índices de asimetría intracromosomal (MCA) e intercromosomal (CVCL) siguiendo lo propuesto por Peruzzi \& Eroglu (2013).

\section{RESULTADOS}

Las siete poblaciones de $A$. diluta analizadas presentan un $2 \mathrm{n}=2 \mathrm{x}=16$ cromosomas. Ambas subespecies presentan una fórmula cromosómica haploide de $3 \mathrm{~m}+1 \mathrm{sm}+1 \mathrm{st}+$ $3 \mathrm{t}$, es decir, tres pares de cromosomas metacéntricos, un par submetacéntrico, un par subtelocéntrico y tres pares telocéntricos (Fig. 1 C-F). Los valores de los índices de asimetría del cariotipo CVCL y MCA y la longitud total diploide de los cromosomas (LTC) evidencian una clara diferencia entre las poblaciones de cada subespecie analizada (Tabla I). La Figura 3 representa el diagrama de dispersión de los datos de los índices CVCL vs MCA (Peruzzi \& Eroglu 2013), donde se puede observar que cada una de las poblaciones dentro de cada subespecie aparecen agrupadas entre si, pero separadas entre las subespecies.

\section{DISCUSIÓN}

Baeza et al. (2010b) realizaron una caracterización del cariotipo en una población de $A$. diluta de la Región de Valparaíso, encontrando que presentaba un cariotipo muy asimétrico y un valor diploide de LTC de 119, $6 \mu \mathrm{m}$, correspondiendo estos valores a los más bajos encontrados en una especie perenne de Alstroemeria en Chile y sólo comparables a los reportes de Jara-Seguel et al. (2004) descritos para A. graminea Phil., única especie anual dentro del género. En el presente trabajo se reportan antecedentes citotaxonómicos en dos nuevas poblaciones de la Región de Valparaíso y los valores encontrados son muy similares a lo informado anteriormente, esto es, para la población 4390, recolectada en el Jardín Botánico Nacional, se describe un LTC de $124 \pm 2,6 \mu \mathrm{m}$ y para la población 4391 , de Punta Curaumilla, este valor fue de 117,8 $\pm 1,2 \mu \mathrm{m}$ (Tabla I). Coincidentemente, las poblaciones 4392 y 4393 de la Región del Maule también presentan valores similares de LTC, $102,2 \pm 4,6 \mu \mathrm{m}$ para la primera y $123,6 \pm 2,8$ para la segunda. Las poblaciones de la Región de Coquimbo presentan valores más altos de LTC que las poblaciones del centro y sur de Chile analizadas, esto es, la población 4376 de Guanaqueros presenta un LTC de 149,0 \pm 9,7 $\mu \mathrm{m}$, la 4412 también proveniente del sector Guanaqueros de 147,4 $\pm 8,5$ $\mu \mathrm{m}$ y la 4415 del Parque Nacional Fray Jorge de $145,8 \pm$ $6,8 \mu \mathrm{m}$. Las poblaciones del norte presentan en promedio más de un $20 \%$ de LTC que las poblaciones de las regiones

TABla I. Características del cariotipo de las subespecies de Alstroemeria diluta. CVCL = Coeficiente de variación del largo de los cromosomas. MCA = Índice de asimetría de la media centromérica de acuerdo a Peruzzi \& Eroglu (2013). SD = Desviación estandar. LTC $=$ Longitud total de los cromosomas.

TABLE I. Karyotype features of the subspecies of Alstroemeria diluta. $\mathrm{CVCL}=$ Coefficient of variation of chromosome length. MCA $=$ Mean centromeric asymmetry index according to Peruzzi \& Eroglu (2013). SD = Standard deviation. LTC $=$ Total chromosome lenght.

\begin{tabular}{lccc} 
TAXA (N DE RECOlECción) & MCA \pm D.S. & CVCL \pm D.S. & LTC $(\mu \mathrm{m}) \pm$ D.S. \\
\hline A. diluta subsp. chrysantha (4376) & $47,4 \pm 1,6$ & $56,2 \pm 3,7$ & $149,0 \pm 9,7$ \\
A. diluta subsp. chrysantha (4412) & $49,0 \pm 5,9$ & $55,1 \pm 3,2$ & $147,4 \pm 8,5$ \\
A. diluta subsp. chrysantha (4415) & $48,0 \pm 1,5$ & $57,0 \pm 4,0$ & $145,8 \pm 6,8$ \\
A. diluta subsp. diluta (4390) & $45,0 \pm 2,4$ & $48,4 \pm 0,6$ & $124,0 \pm 2,6$ \\
A. diluta subsp. diluta (4391) & $44,5 \pm 0,1$ & $48,4 \pm 0,3$ & $117,8 \pm 1,2$ \\
A. diluta subsp. diluta (4392) & $44,7 \pm 0,5$ & $46,5 \pm 1,2$ & $102,2 \pm 4,6$ \\
A. diluta subsp. diluta (4393) & $44,8 \pm 0,6$ & $50,3 \pm 1,4$ & $123,6 \pm 2,8$ \\
\hline
\end{tabular}



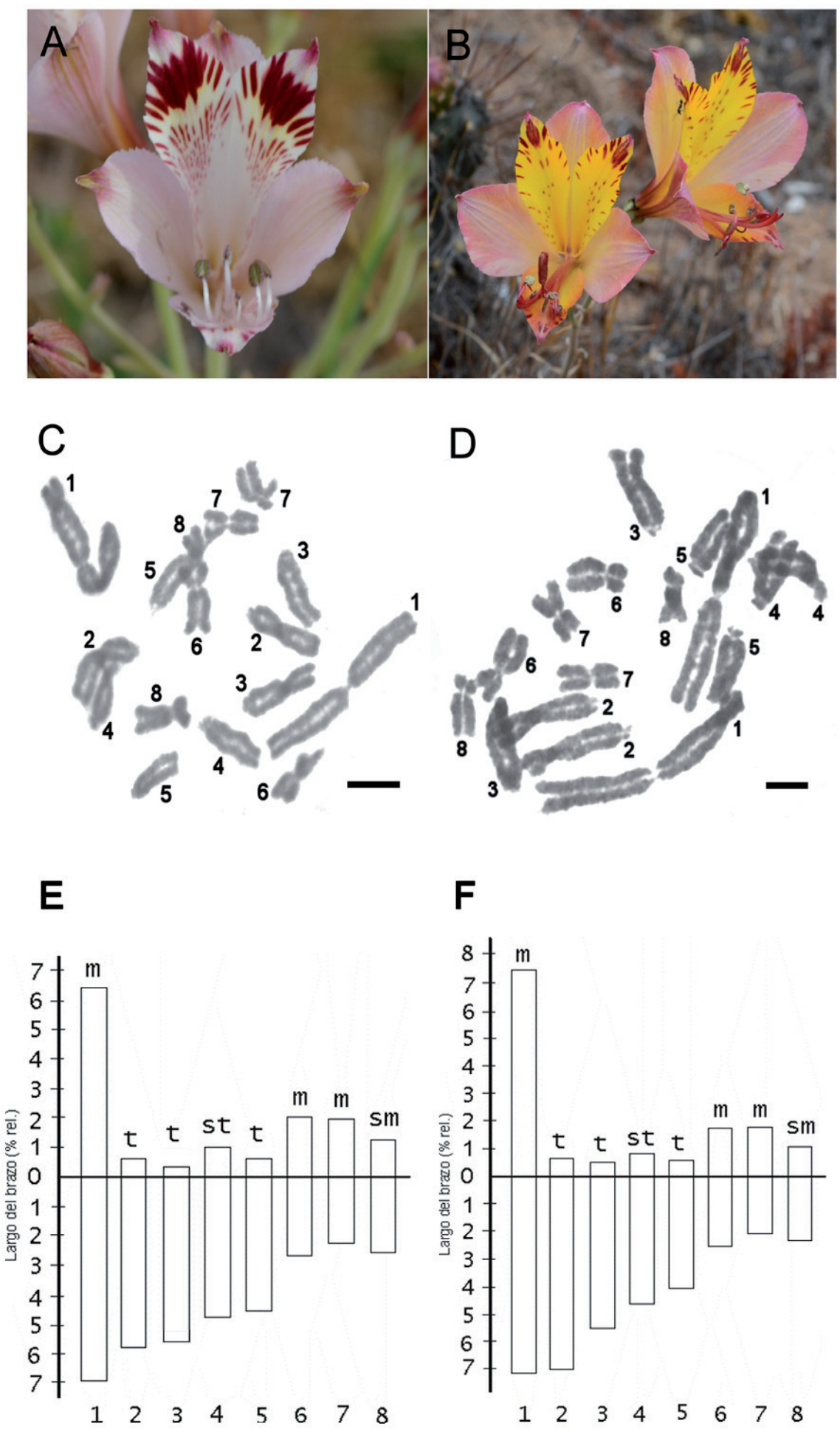

Figura 1. Fotografías, placas metafásicas e idiogramas de Alstroemeria diluta. A. Fotografía de A. diluta subsp. diluta. B. Fotografía de $A$. diluta subsp. chrysantha. C. Placa metafásica de A. diluta subsp. diluta (4392). D. Placa metafásica de A. diluta subsp. chrysantha (4376). E. Idiograma de $A$. diluta subsp. diluta (4392). F. Idiograma de A. diluta subsp. chrysantha (4376). La escala corresponde a $5 \mu$ m.

Figure 1. Photographies, metaphasic plates, and idiogramms of Alstroemeria diluta. A. Photographie of A. diluta subsp. diluta. B. Photographie of $A$. diluta subsp. chrysantha. C. Methaphasic plate of A. diluta subsp. diluta (4392). D. Methaphasic plate of $A$. diluta subsp. chrysantha (4376). E. Idiogram of $A$. diluta subsp. diluta (4392). F. Idiogram of $A$. diluta subsp. chrysantha $(4376)$. Bar $=5 \mu \mathrm{m}$. 


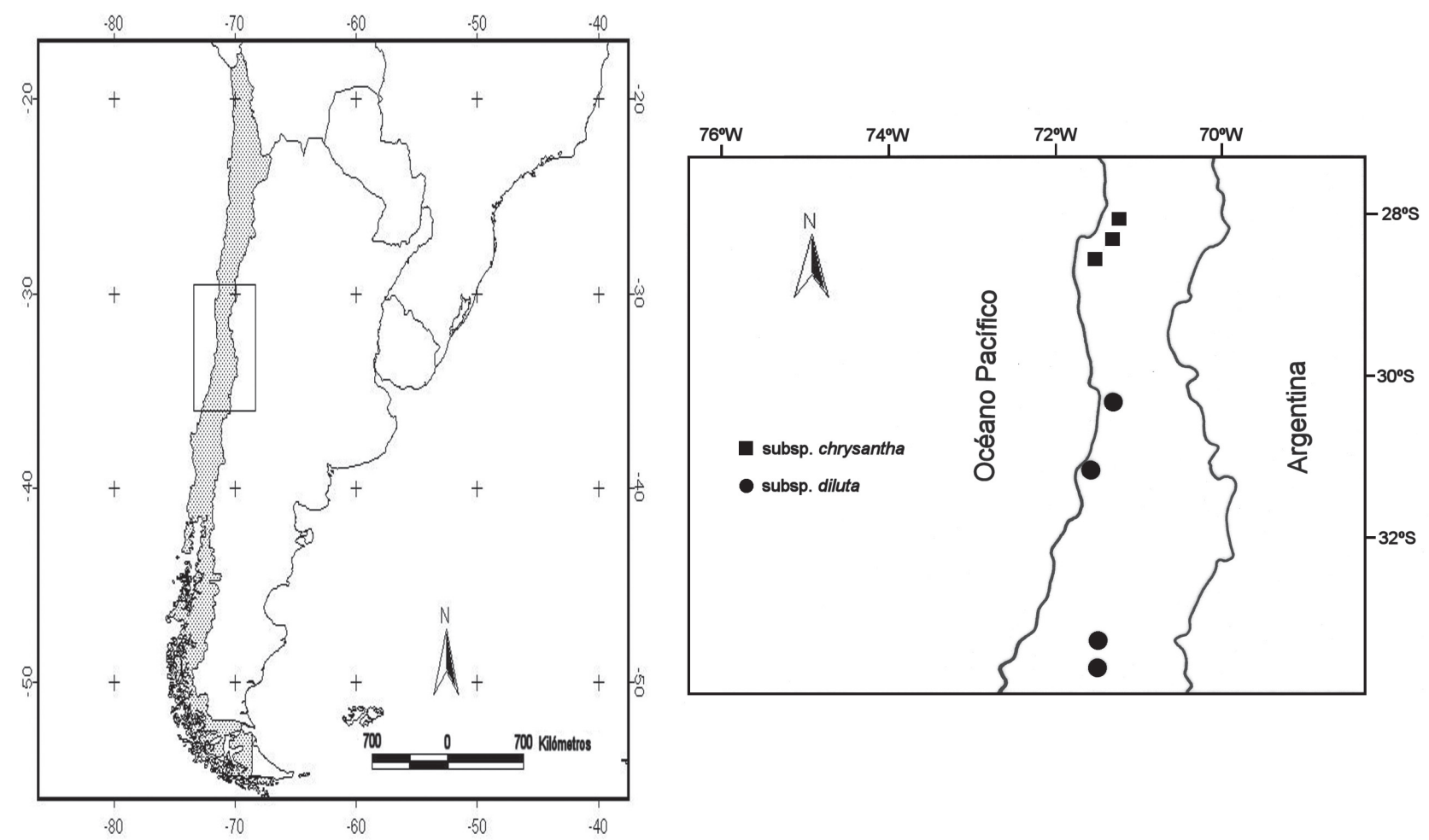

FiguRA 2. Distribución geográfica de las 7 poblaciones del complejo Alstroemeria diluta analizadas

FIGURE 2. Geographic distribution of the 7 analyzed populations of the Alstroemeria diluta complex.

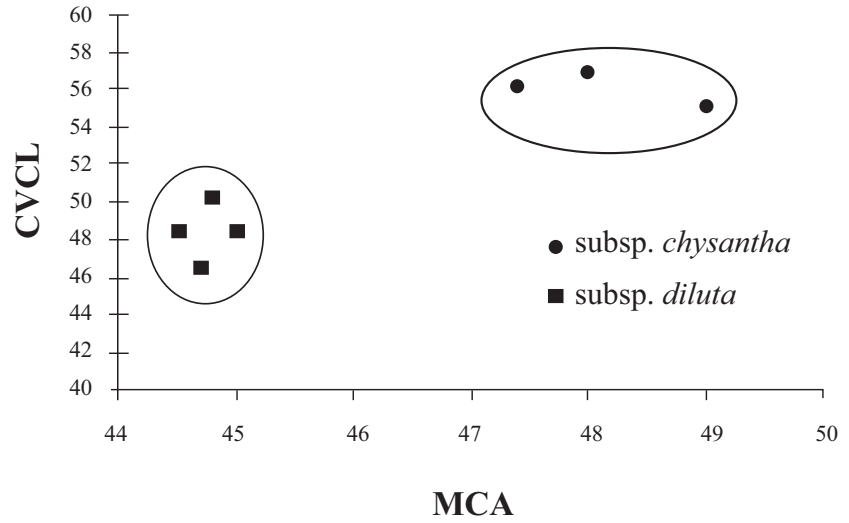

FiguRA 3. Diagrama de dispersión entre las poblaciones de las subespecies de Alstroemeria diluta usando valores de MCA vs CVCL.

FigURE 3. Scatter plot among populations of Alstroemeria diluta subspecies using values of MCA vs CVCL. de Valparaíso y del Maule. Sin embargo, la morfología de los cromosomas en todas las poblaciones analizadas citológicamente es idéntica, es decir, no existen diferencias en los tipos de cromosomas (Fig. 1 C-F); la diferencia radica exclusivamente en el LTC de las poblaciones estudiadas.

En el diagrama de dispersión MCA vs CVCL (Fig. 3), se puede observar que las 4 poblaciones de la subsp. diluta se agrupan entre ellas y las 3 poblaciones de la subsp. chrysantha también lo hacen entre ellas. Además, se puede apreciar una importante separación entre ambas subespecies, siendo el índice intracromosomal (MCA) el que más contribuye a dicha separación. Este índice cromosómico está estrechamente relacionado con la longitud de los cromosomas (Peruzzi \& Eroglu 2013). De esta forma, las poblaciones de la Región de Valparaíso pertenecerían a la subsp. diluta y no a la subsp. chrysantha, lo que permitiría extender la distribución de esta subespecie hasta la Región de Valparaíso, restringiendo la distribución de la subsp. chrysantha al sur de las Regiones de Atacama y Coquimbo. Otra situación interesante es que las poblaciones analizadas de la subsp. chrysantha se encontraron creciendo en suelos arenosos, en cambio, las poblaciones de la subsp. diluta lo hacían en suelos duros y extremadamente compactos. Muñoz \& Moreira (2003) extienden la distribución de la 
subsp. chrysantha desde la Región de Atacama hasta la Región de Valparaíso e indican dos localidades de la subsp. diluta en la Región de O’Higgins. Hoffmann et al. (2015) reconocen $A$. diluta subsp. diluta y $A$. x chrysantha ( $A$. diluta subsp. chrysantha), indicando a este último taxón como un híbrido natural que se distribuye en la zona norte centro de Chile, desde los llanos costeros de la Región de Atacama hasta Curaumilla, en la Región de Valparaíso. Estudios de colorimetría en curso (Finot, comunicación personal) sugieren que las poblaciones de la Región de Valparaíso están mucho más relacionadas a las poblaciones de la Región del Maule que a las poblaciones de la Región de Coquimbo, lo que es coincidente con los datos obtenidos en el diagrama de dispersión MCA vs CVCL. La distribución de las poblaciones en el espacio CIELab (Commision Internationale de l'Eclairage 1978) separan las poblaciones de las subespecies chrysantha y diluta tanto por los valores de C (Croma o saturación), como de $\mathrm{h}^{\mathrm{o}}$ (hue, tono), a* (verde-rojo) y $\mathrm{b}^{*}$ (azul-amarillo) tanto para los tépalos externos como internos superiores e interno inferior. Los valores de $b^{*}$ alcanzan valores mucho mayores en la subsp. chrysantha debido al color amarillo en comparación con la subsp. diluta que alcanza valores de $b^{*}$ muy bajos. Por su parte, los valores de $\mathrm{a}^{*}$ son menores en la subsp. chrysantha en comparación con la subsp. diluta.

En definitiva, ambas subespecies de $A$. diluta presentan los mismos cromosomas, es decir, la arquitectura de los cromosomas no es un carácter que permita diferenciar las subespecies y apoyar la hipótesis propuesta por Bayer (1987). Sin embargo, la longitud de los cromosomas (LTC) las permite diferenciar claramente, por lo tanto, este es un buen carácter citotaxonómico que apoya lo propuesto por Bayer (1987) y Muñoz \& Moreira (2003).

\section{AGRADECIMIENTOS}

Trabajo financiado por proyecto Fondecyt $N^{\circ} 1130349$. También se agradece el apoyo prestado por Conaf, por permitir recolectar material en el Parque Nacional Fray Jorge. Mis agradecimientos al Departamento de Botánica de la Universidad de Concepción, por todas las facilidades otorgadas.

\section{BIBLIOGRAFÍA}

Amigo, J. \& C. Ramírez. 1998. A bioclimatic classification of Chile: Woodland communities in the temperate zone. Plant Ecology 136: 9-26.
Baeza, C., E. Ruiz \& M. Negritto. 2010a. Comparative karyotypic analysis in the Alstroemeria hookeri Lodd. (Alstroemeriaceae) complex sensu Bayer (1987). Genetics and Molecular Biology 33: 119-124.

Baeza, C., E. Ruiz \& P. Novoa. 2010b. Karyotype of Alstroemeria diluta Ehr. Bayer subsp. chrysantha (Alstroemeriaceae). Chilean Journal of Agricultural Research 70: 667-669.

BAezA, C. \& E. RuIz. 2011. Alstroemeria hookeri Lodd. subsp. sansebastiana C.M. Baeza \& E. Ruiz, nueva para la flora de Chile. Gayana Botánica 68: 313-315.

Baeza, C., V. Finot \& E. Ruiz. 2015a. Comparative karyotype analysis of populations in the Alstroemeria presliana Herbert (Alstroemeriaceae) complex in Chile. Genetics and Molecular Biology 38: 199-204.

Baeza, C., V. Finot, E. Ruiz, P. Carrasco, P. Novoa, T. Stuessy \& A. GonzÁlez. 2015b. Comparative karyotypic analysis and cytotaxonomy in the Alstroemeria ligtu L. (Alstroemeriaceae) complex of Chile. Brazilian Journal of Botany. 39(1):305-3013.

BAYER, E. 1987. Die Gattung Alstroemeria in Chile. Mitteilungen der Botanischen Staatssamlung München 24: 1-362.

Cajas, D., C. Baeza, E. Ruiz \& M. Negritto. 2009. Cytogenetics analysis in populations of Alstroemeria hookeri Lodd. subsp. hookeri (Alstroemeriaceae) from Bío-Bío Region, Chile. Gayana Botánica 66: 117-126.

Commision Internationale de L'Eclairage (CIE). 1978. Recommendations on uniform color spaces: color difference equations, psychometric color terms. Publ. CIE 15, Suppl. 2, Paris.

Hoffmann, A., J. Watson \& A. Flores. 2015. Flora silvestre de Chile. Cuando el desierto florece. Volumen 1. Monocotiledóneas y otros taxones. Fundación Claudio Gay. Salesianos impresores S.A. 261 pp.

Jara-Seguel, P., C. Palma-Rojas \& E. Brand. 2004. Karyotype and C-bands in the annual Inca Lily Alstroemeria graminea. Belgian Journal of Botany 137: 199-204.

Muñoz, M. \& A. Moreira. 2003. Alstroemerias de Chile. Diversidad, distribución y conservación. Taller La Era, Santiago de Chile. 140 pp.

Negritto, M., C. Baeza, E. Ruiz \& P. Novoa. 2015. Alstroemeria marticorenae (Alstroemeriaceae), a new species from central Chile. Systematic Botany 40: 69-74.

Peruzzi, L. \& H. Eroglu. 2013. Karyotype asymmetry: again, how to measure and what to measure? Comparative Cytogenetics 7: 1-9.

Reeves, A. 2001. MicroMeasure: a new computer program for the collection and analysis of cytogenetic data. Genome 44: 239-443.

Vergara, O., V. Jerez \& E. Parra. 2006. Diversidad y patrones de distribución de coleópteros en la Región del Bíobío, Chile: Una aproximación preliminar para la conservación de la diversidad. Revista Chilena de Historia Natural 79: 369-388.

Recibido: 02.12.15

Aceptado: 01.07.16 